Culture et histoire dans l'espace roman

4 | 2010

Les représentations du corps dans la litterature latinoaméricaine

\title{
José Watanabe: cuerpo, patria, naturaleza
}

Luis Fernando Jara

\section{(2) OpenEdition \\ Journals}

Edición electrónica

URL: https://journals.openedition.org/cher/8177

DOI: $10.4000 /$ cher.8177

ISSN: 2803-5992

Editor

Presses universitaires de Strasbourg

\section{Edición impresa}

Fecha de publicación: 30 junio 2010

Paginación: 261-272

ISBN: 978-2-35410-007-0

ISSN: 1968-035X

Referencia electrónica

Luis Fernando Jara, «José Watanabe: cuerpo, patria, naturaleza», reCHERches [En línea], 4 | 2010,

Publicado el 15 diciembre 2021, consultado el 28 enero 2022. URL: http://journals.openedition.org/ cher/8177; DOI: https://doi.org/10.4000/cher.8177

\section{(c) (i) (2)}

Ce(tte) œuvre est mise à disposition selon les termes de la Licence Creative Commons Attribution Pas d'Utilisation Commerciale - Partage dans les Mêmes Conditions 4.0 International. 


\title{
José Watanabe: cuerpo, patria, naturaleza
}

\author{
Luis Fernando Jara \\ Université de Toulouse - Le Mirail / Pontificia Universidad Católica del Perú
}

Tosé Watanabe (1946-2007) es uno de los principales poetas peruanos en español hasta alcanzar un lugar privilegiado. Muchos datos confirman esta importancia gradual, como la recepción que ha tenido, entre la crítica española, la reciente publicación de su poesía completa (Watanabe 2008). Una de las primeras reseñas reconoce el valor de esta escritura:

No cabe duda de que el conjunto de la producción de Watanabe constituye una de las experiencias más renovadoras no sólo de la poesía peruana [...], sino de la poesía hispanoamericana - incluyendo la española - reciente (Marco 2008).

Uno de los rasgos más distintivos de la poesía de Watanabe es la sistemática presencia de dos temas: el cuerpo y Laredo, el pueblo donde nació. Esta recurrencia cobra especial interés si se tiene en cuenta unas declaraciones del poeta en las que vincula esos contenidos con el concepto de patria:

La primera patria es el cuerpo; la segunda, el lugar donde uno ha nacido, sea el pueblo o el barrio. Después no hay más patria. Hay un verso de Gonzalo Rojas que dice: «Sí, soy ciudadano, pero ¿̇e dónde?». Siempre repito ese verso. Yo soy ciudadano de mi cuerpo [...]. (Ordaz 2005: 51).

Antes hemos aludido al rol decisivo que tiene Laredo en la poesía de Watanabe (Jara 2007). Ahora, queremos clarificar el parentesco entre la patria y el cuerpo; de qué modo éste puede representar o sustituir a aquél. 
Partimos del supuesto de que en Watanabe es crucial la noción de un "antes" de la patria, identificada con la naturaleza, una suerte de patria primordial. Esto explica que en su poesía prevalezca una visión panteísta del mundo, como veremos más adelante.

\section{La noción de patria}

A la luz de la cita anterior, parece evidente que Watanabe no define el concepto de patria como lo dictan el sentido común y los diccionarios. El DRAE (Diccionario de la Real Academia Española) propone dos definiciones para el concepto de "patria":

1. f. Tierra natal o adoptiva ordenada como nación, a la que se siente ligado el ser humano por vínculos jurídicos, históricos y afectivos.

2. f. Lugar, ciudad o país en que se ha nacido.

La «tierra natal ordenada como nación» es el Perú; con el que, según se deduce de la cita, Watanabe no se siente ligado por ningún tipo de vínculo. Si la cultura es producto en gran parte de procesos inconscientes, la identidad (nacional) supone un vínculo de pertenencia necesariamente consciente, fundada en oposiciones simbólicas (Cuche 1996: 82). Para Watanabe, su cuerpo y Laredo son las únicas patrias que reconoce: «Después no hay más patria», afirma con contundencia. Su idea de patria está más cerca de la segunda definición que propone el DRAE, pues coincide con la segunda que propone Watanabe: «lugar donde uno ha nacido», lo que suele llamarse la «patria chica».

Su primera noción de patria es, sin embargo, mucho más restrictiva, sutil y personal, pues implica relaciones más próximas, en términos físicos y afectivos: las correspondencias que entreteje el hombre con su cuerpo. ¿De dónde proviene esta particular concepción de patria? Presumimos que en su origen hay dos experiencias vitales determinantes: su condición nikkei y la enfermedad que padeció, como veremos a continuación.

\section{La condición nikkei}

Quizás sorprenda mencionar el mestizaje como rasgo distintivo de un poeta peruano si se tiene en cuenta que, por definición, es el carácter que define por excelencia a toda la cultura peruana. Habría que considerar, sin embargo, los distintos tipos de mestizaje que se han dado al interior de esta cultura. No nos vamos a detener aquí en una explicación detallada de esos 
procesos. Solo queremos enfatizar un hecho: Watanabe es producto de uno de los mestizajes más idiosincrásicos que se han dado en el conjunto de mixturas étnicas en el Perú, pues es hijo de una campesina andina y de un inmigrante japonés ${ }^{1}$.

¿En qué radica la idiosincracia de este mestizaje? En el hecho de que involucra, en una de sus filiaciones, a un inmigrante japonés. Durante mucho tiempo, en el Perú, esa pertenencia étnica implicó una sospecha que involucraba también a sus descendientes (los llamados nikkeis): su no pertenencia a la cultura peruana, desconfianza que no se hacía extensiva a los descendientes de otras etnias o culturas ${ }^{2}$. Esa suspicacia se tradujo, a veces, en un explícito rechazo. En los años treinta del siglo XX,

la comunidad nikkei es punto de ataques que tienen como explicación puntos irracionales, como el ser agentes de enfermedades, el considerarse una raza extraña a la que no debieran abrírsele las puertas con tanta facilidad, el ser «feos soldados del Imperio», y otras cuestiones que expresan un rechazo a la comunidad, un rechazo que usualmente se conoce como xenofobia (Takashi Martínez 2009).

Los descendientes de japoneses fueron víctimas de un «racismo institucional», es decir, las manifestaciones racistas no eran hechos aislados motivados por prejuicios, sino el resultado de una estructura social sustentada en reglas, de procedimientos y prácticas institucionalizadas por los aparatos burocráticos y las instituciones formales. Esos comportamientos xenofóbicos se multiplicaron y fueron más graves aún durante la Segunda Guerra Mundial ${ }^{3}$.

1 La inmigración japonesa comenzó en 1899 y se concentró en la costa norte del Perú. Su padre llegó de Okiyama en 1916 y se instaló como agricultor en los latifundios azucareros próximos a Trujillo. Su madre venía de Otuzco, un pueblo de la serranía de La Libertad, instalado en el corazón del ande peruano. La cultura japonesa de su padre y la cultura andina de su madre coincidieron en Laredo, donde se instaló y creció la familia Watanabe Varas y su prole numerosa de once hijos.

2 Efectivamente, "ha habido momentos de la historia en el que la sociedad peruana ha sido un tanto arisca con los inmigrantes de procedencia extraña a la europea, que es la cultura con la cual, desde tiempos coloniales, ha estado más compenetrada por razones históricas», Takahashi Martinez, Yukiko, "Ciudadanos de origen japonés en el Perú durante la Segunda Guerra Mundial: una revisión de los debates actuales», http://www. discovernikkei.org. Consultado el 08/03/2009.

3 «Las cifras son unánimes, entre 2000 y 2300 nikkeis fueron deportados de América Latina a Estados Unidos, 1771 de los cuales, llegaban de Perú. El destino, campos de reubicación principalmente al sur, en California y Texas (Manzanar, Crystal City, Seagoville)», (Takashi Martínez 2009). 
El propio Watanabe recuerda que, cuando iba a la escuela, los compañeros de clase solían plantearle la siguiente pregunta: «¿si el Perú y Japón se enfrentasen en una guerra, a favor de qué país estarías tú?»» ${ }^{4}$ Es importante decir que el niño que entonces era Watanabe defendía con ahínco su pertenencia al Perú. Buena parte de su vida escolar la pasó intentando convencer a los "otros" de que él era un peruano como ellos, como cualquiera.

No sé si sus condiscípulos de entonces terminaron persuadidos de la identidad peruana de Watanabe; conjeturamos, en cambio, que esa insistente pregunta contribuyó a erosionar las convicciones que sustentaban su temprana defensa de la identidad nacional ${ }^{5}$. Nos parece pertinente aclarar que la condición nikkei no implica necesariamente un debilitamiento de la relación con la patria o una negación automática de la identidad nacional. Las relaciones peculiares que un individuo establece con su patria son producto de un conjunto de factores.

El desarraigo, elegido o impuesto, puede ser un agente determinante en esa relación. $\mathrm{Si}$, como en Watanabe, el cuerpo es el lugar donde reposa la ciudadanía - «soy ciudadano de mi cuerpo» - y el elemento tangible que se lleva de un lugar a otro, entonces el desplazamiento labra también la identidad, como ocurrió con sus padres:

$[\ldots]$

25 yo soy el que voy, y salto

para que las inmensidades

me vean. Mírenme

trayendo en mis brazos mi propio cuerpo

[...] («Regresando al Perú en barco», Cosas del cuerpo, p. 112)

Watanabe ha aludido en diversos textos a su condición nikkei. «El lenguado», poema liminar de Cosas del cuerpo (1999), uno de los más bellos textos de Watanabe, empieza con estos versos:

Soy

lo gris contra lo gris. Mi vida

depende de copiar incansablemente

el color de la arena,

4 Entrevista que efectué a José Watanabe entre el 15 y 18 de abril de 2003.

5 El ambiente familiar tampoco era propicio para alentar o consolidar una conciencia de identidad nacional; todo lo contrario, como lo recuerda Watanabe: «Los hijos de los inmigrantes japoneses escuchamos en nuestra infancia que algún día toda la familia iría a Japón» (Watanabe 2003: 145). 
5 pero ese truco sutil

que me permite comer y burlar enemigos

me ha deformado. He perdido la simetría

de los animales bellos

[...]

Estos versos hablan de adaptaciones que mudan las apariencias y que fuerzan ciertos sacrificios, pues la supervivencia implica una pérdida, la de «la simetría de los animales bellos». ¿Puede plantearse esta analogía como una reflexión sobre la identidad nikkéi, sobre la pulsión de una necesidad de acomodación, de conciliación? Watanabe termina el poema con una alusión al sueño:

22 A veces sueño que me expando

y ondulo como una llanura, sereno y sin miedo, y más grande

que los más grandes. Yo soy entonces

toda la arena, todo el vasto fondo marino.

(«El lenguado», Cosas del cuerpo, p. 936).

Es sintomático que el primer poema de un libro cuyo título alude explícitamente al cuerpo empiece con una parábola sobre la identidad. $Y$, en relación con la pregunta anterior, otra pregunta es pertinente: ¿son estos últimos versos una metáfora de la actividad artística como espacio de libertad, como medio que anula las fronteras, las identidades nacionales?

\section{La enfermedad}

La otra experiencia determinante en la vida de Watanabe es la enfermedad. Una parte considerable de su obra poética se refiere a su prolongada estancia en un hospital alemán para recuperarse de una grave dolencia que lo llevó al borde de la muerte. Esta difícil experiencia le confirma y le agudiza la conciencia de la fragilidad del cuerpo, que ya había intuido ${ }^{7}$. Su primer libro, Álbum de familia (1971), alude a las difíciles condiciones en que vivía la gente de su pueblo:

Ahora no hay lugar a discusión ni defensa.

La peste tenía su oficio.

6 Las páginas corresponden a Watanabe: 2003.

7 Sobre todo, el oficio atroz e imperturbable de la peste, negrísimo heraldo de la muerte, que se ensañaba en sociedades precarias como Laredo: «Mi pueblo era muy modesto y venían enfermedades, peste bubónica, meningitis, tanto así que mis dos hermanos anteriores a mí murieron de meningitis. Estábamos expuestos a las pestes y no teníamos medios para curarnos», (Ordaz 2005: 51). 
Fue duro verte rodar como una semilla.

Yo sobrevivo entre los muertos.

Caminamos por los pasillos como en esas silenciosas y vastas posadas.

Respiramos el deseo de huir sin cancelar la cuenta.

(«Informe para mi hermano muerto en la infancia», Álbum de familia, p. 20)

La enfermedad aludida le revela también el lado atroz del sufrimiento. Watanabe es consciente de que el dolor tiene un carácter privativo y una existencia concreta, aunque nadie lo advierta y nada sea su signo. El dolor habita en el lugar más íntimo del cuerpo, donde establecen una feroz convivencia, como lo dejan entrever los siguientes versos:

[...]

5 Una enfermera cruza el jardín, ninguna

flor anuncia mi dolor. El dolor solo está

en los confines de la carne que aún me resta.

[...]

(«Cielo de hospital», Cosas del cuerpo, p. 99)

Pero, si en el cuerpo se sufre, Watanabe quiere creer que en el cuerpo también se vence al dolor. Así lo creía la gente de su pueblo natal, que se enfrentaba a las enfermedades con sabiduría popular, es decir, con los conocimientos ancestrales transmitidos de generacion en generacion, que se sustentan en una nocion de realidad y una vision de mundo alternativas a las nociones gobernadas por la lógica y la ciencia occidentales:

El cascarón liso del huevo

sostenido en el cuenco de la mano materna

resbalada por el cuerpo del hijo, allá en el norte.

Eso vi:

5 Una mujer más elemental que tú

espantando a la muerte con ritos caseros, cantando

con un huevo en la mano, sacerdotisa

más modesta no he visto.

Yo la miraba desgranar sobre su regazo

10 los maíces de la comida

mientras el perro callejero se disolvía en el relente del sol

lamiendo

el dolor arrojado a la tierra

junto con el huevo del milagro.

$[\ldots]$ 
El único valor era vivir.

[...]

20 y las gallinas alineaban en su vientre sus santas ovas

y mi madre esperaba nuevamente el más fresco huevo

con un convencimiento:

La vida es física.

Y con ese convencimiento frotaba el huevo contra mi cuerpo

25 y así podía vencer.

En ese mundo quieto y seguro fui curado para siempre.

En mí se harán todos los milagros.

[...]

(«La cura», Historia Natural, p. 70)

El convencimiento de que "la vida es física», cuya enunciación destaca en la estructura del poema, pues constituye un verso independiente, lleva a la madre - y a la gente con fuerte ascendencia campesina o rural, como la que habita el Laredo ${ }^{8}$ donde está contextualizado el poema - a imaginar que todo lo relacionado con el cuerpo es físico; por lo tanto, también las enfermedades 9 . Si los males corporales tienen existencia concreta entonces tienen una forma, asible y perceptible para el ojo humano. El huevo es el medio a través del cual se aprehende y se vence a la enfermedad ${ }^{10}$.

8 Laredo era, entonces, un pequeño y humilde pueblo de trabajadores de las haciendas azucareras próximas a él; un pueblo detenido en el tiempo, habitado por mitos y fantasmas. Las gentes que formaron ese pueblo cargaban cada una también su mitología personal.

9 Puede ser interesante advertir la relacion fónica que es factible establecer entre la preeminencia de las mismas vocales fuertes en la palabras cruciales de los versos citados: «fresco», «huevo», «convencimiento», «cuerpo» y «quieto». Son términos importantes porque constituyen el núcleo del sentido del poema: el convencimiento de los efectos curativos del huevo fresco sobre el cuerpo efectivamente curaba, lo que generaba la sensación de vivir en un mundo quieto (y seguro). Además, la repetición de la pareja de vocales «e-o» semeja un eco que resuena en la memoria del yo poético y que viene desde la infancia.

10 Esta es una creencia extendida entre las gentes que conservan costumbres del campo o de ámbitos rurales. Al ser frotado contra el cuerpo, el huevo (en otros rituales puede ser el cuy, que tiene las mismas propiedades curativas) absorbe la enfermedad, el mal o la dolencia (el susto o el «mal del ojo» suelen ser las que más convocan el auxilio del huevo) que es perceptible cuando se rompe la cáscara y se vierte su contenido dentro de un vaso. De acuerdo con la costumbre, allí puede verse el origen de la dolencia o el órgano al que ha afectado. 


\section{La naturaleza: patria primigenia}

Pero, Watanabe, en cuya conciencia el mito todavía no está enraizado, sabe que «en ese mundo quieto y seguro [no] fue curado para siempre» y que en él tampoco «se harán todos los milagros». La enfermedad habita su cuerpo y hace estragos en él. Esa experiencia límite, en la que se convive con el dolor y se está en el abismo de la muerte, cambia radicalmente en Watanabe su concepción del cuerpo y de la condición humana. Leamos el siguiente poema:

Una delgada columna de sangre desciende desde una bolsa de polietileno hasta la vena mayor de mi mano. ¿Qué otro corazón la impulsaba antes, qué otro corazón más vigoroso y espléndido que el mío, lento y trémulo? Esta sangre que me reconforta es anónima. Puede ser de cualquiera. Yo voy (o iba) para ser misántropo y no quiero una deuda sospechada en todos los hombres. ¿Cuál es el nombre de mi dador? A ese solo y preciso hombre le debo agradecimiento. Sin embargo, la sangre que está entrando en mi cuerpo me corrige. Habla, sin retórica, de una fraternidad más vasta. Dice que viene de parte de todos, que la reciba como un envío de la especie.

(«El envío», El huso de la palabra, p. 54)

El yo poético reconoce una "deuda" con la especie humana. La sangre anónima que reanima el cuerpo del enfermo anula la introversión a la que éste tendía y le hace tomar conciencia de la solidaridad de la especie ${ }^{11}$. Otra idea crucial se le revela; otra conciencia de la pertenencia, la noción de un antes de la patria:

[...]

Tendido, tu cuerpo suena sus tripas y te recuerda que aún te quedan tus humildes voces

10 vegetativas.

$[\ldots]$

Callada tu mente y su prestigioso trabajo,

15 descubres, en el peligroso borde, que tu cuerpo es inteligente

y que es tuyo y de todos. Todo cuerpo es tótem.

Levántate y muestra tu desnudez al alba que ya empieza.

A las 7 los cirujanos te abrirán el pecho con sus escalpelos.

20 No morirás: tus voces vegetativas siguen sonando

y ya son (y ya eres) parte del rumor panteísta que viene del bosque

11 Esa «fraternidad más vasta» a la que el yo poético le debe la vida vincula este poema con «Masa», de Vallejo, pues en ese texto también es la especie humana la generadora de vida y también hay un signo de agradecimiento en el abrazo emocionado que da el cadáver resucitado. 
$\mathrm{y}$, al parecer, de un alba más remota.

(«Como el peje-sapo», Elogio del refrenamiento, p. 52)

Watanabe parece atribuirle al cuerpo una conciencia y, desde ella, una reflexión radical que descubre sus raíces primigenias. La relación del cuerpo con la naturaleza no es una relación intelectual o de posesión, sino una relación viviente y vivida. El cuerpo poseería lo que Merleau-Ponty llama «la ciencia implícita y sedimentada del mundo» (citado por Matos Dias 2001: 47). y sería la mediación del sujeto consigo mismo («Cielo de hospital»), con los otros («El envío»), con el orden natural («Como el pejesapo»).

Por otro lado, "Todo cuerpo es tótem», es decir, cualquier cuerpo es una entidad de la naturaleza que puede asumir el rol de emblema protector de la tribu o del individuo, o ser asumido como ascendiente o progenitor. $\mathrm{Si}$ todo cuerpo es tótem, entonces le pertenece a la naturaleza, tiende a ella. Su encuentro supone una confluencia de saberes - los del cuerpo y los de la naturaleza - que la relación macrocosmo y microcosmo había ya anunciado.

En los versos leídos, la suspensión de la conciencia, del razonamiento, hace aflorar el primigenio e instintivo lenguaje del cuerpo (las «voces vegetativas») que, como parte de un «rumor panteísta», se vincula con la naturaleza. Se establecería, entonces, el parentesco entre «l'être de la terre et celui de mon corps» (Matos Dias 2001: 169), como afirma Merleau-Ponty, quien habla del ser de la tierra - de la naturaleza - como de una «Chair Universelle» (Matos Dias 2001: 169).

El yo poético quiere recobrar ese vínculo con el orden natural. La enfermedad le ha revelado «el peligroso borde», la fragilidad de la vida y del cuerpo, y la posibilidad de diluirse en la nada. Entonces, aparece el deseo de ser algo más que un órgano tocado por el tacto inexorable del tiempo. El yo poético revela la voluntad de trascender la corporeidad, de diluirse en el orden natural, de ser uno con la naturaleza, «el alba más remota» de donde se viene, el espacio que engloba todo lugar, todo tiempo y todo ser:

Otra vez es tiempo de ir a la montaña a buscar una cueva para hibernar.

[...]

Allí está, hermosa e inocente entre la neblina, y yo entro

15 en su perfecta indiferencia

y me ovillo entregado a la idea de ser de otra sustancia.

He venido por enésima vez a fingir mi resurrección. 
En este mundo pétreo

nadie se alegrará con mi despertar. Estaré yo solo

20 y me tocaré

y si mi cuerpo sigue siendo la parte blanda de la montaña

sabré

que aún no soy la montaña.

(«Animal de invierno», Cosas del cuerpo, p. 97)

Se busca insistentemente la comunión con el orden natural por medio de una añorada y anhelada relación uterina ${ }^{12}$. El sujeto poético se "ovilla» en la «cueva» de la «montaña» - como un feto en el útero de la madre - buscando la «resurrección», es decir, un nuevo nacimiento, que implicará ser de la misma sustancia imperecedera y dura de la montaña. Es un renacimiento fingido, ciertamente; el verdadero ocurrirá luego de que el cuerpo revele sus «últimas noticias»:

Ésta es tu última noticia, cuerpo:

una radiografía de tus pulmones, brumas

inquietantes, manchas de musgo sobre la nieve sucia.

La tierra espera que algún día

todos los órganos, como los perros, la husmeen

buscando la yerba benéfica. Tus pulmones,

entre hojas sedosas,

lucirán sanos y tersos como recién nacidos

y concertarán con un joven buey

el ritmo amplio de su respiración. Al fondo

habrá un cielo luminoso y ninguna sombra,

sobre todo ninguna sombra aciaga.

(«Última noticia», Banderas detrás de la niebla, p. 24)

Este es uno de los últimos poemas escritos por Watanabe, publicado pocos meses antes de morir. Aquí insiste en este deseo de fundir el cuerpo

12 Esta relación es análoga a la que se establece en el mundo andino entre el hombre y la tierra, a la que se denomina "pachamama», es decir, "madre tierra». Por su filiación materna, Watanabe no es ajeno a la visión andina sobre la tierra y la naturaleza. La relación "maternal" y la visión panteísta es más clara aun en los versos siguientes:

El sol era nuestra leona.

Un viento cálido me envuelve siendo aquí, en Baja Sajonia,

invierno:

es la imagen creando su espacio en mi cuerpo enfermo,

es el sol que me husmea como hijo falto,

allá en el norte de mi país,

donde me enseñó a caminar empujándome con el hocico.

(«Nuestra leona», El huso de la palabra, p. 53) 
con los elementos de la naturaleza, con la esperanza de una resurrección ya no fingida. Si hay una utopía poética en Watanabe ésta consiste en habitar ese locus amenus y en formar parte definitivamente del «rumor panteísta». Se trataría, en realidad, de un paraíso recobrado, en la medida en que morir supone volver a la tierra, al armonioso orden natural.

Una identidad nacional cuestionada permanentemente y una temprana conciencia de la precariedad de la vida y del cuerpo, agudizada por la enfermedad, han conducido casi de modo inexorable a una reflexión poética en cuyo centro palpita el cuerpo y sus cosas. A pesar de su fragilidad, o precisamente por ella, la conciencia de que «la vida es física» ha hecho del cuerpo, materia táctil y sensible, el «lugar" por excelencia para definir una identidad, la patria inmediata. La impronta ineludible del cuerpo convierte a la poesía de Watanabe en:

una operación más del cuerpo. Es una actividad [la poética] que proviene de la entraña, que se confunde con la sangre. Por eso sus versos magníficos poseen el ritmo de una corazonada, la acritud del hígado, la naturalidad de una respiración ${ }^{13}$.

Y una vez más, si «la vida es física”, el cuerpo deviene un asunto de todos los días. El título de uno de sus libros (Cosas del cuerpo, publicado en 1999) y el epígrafe que precede a los poemas - versos del poeta latino Marcial-son más que elocuentes del rol crucial que tiene el cuerpo en la cotidianidad y de la importancia de tenerlo siempre presente:

Que se hable de los dioses

por los siglos de los siglos,

pero del cuerpo y de las cosas del cuerpo

acordémonos todos los días.

La elección de estos versos como epígrafe (que podrían ser considerados el arte poética de Watanabe) y el sentido de una parte importante de su obra revelan que el poeta laredino suscribe la recomendación de Marcial. El cuerpo es, entonces, presencia y experiencia cotidiana; pero en él está también latente, en tanto tótem, la posibilidad de la trascendencia, un espacio sin tiempo, la añorada resurrección en la naturaleza: la patria primordial.

13 López Estrada, Esperanza, Babelia, suplemento cultural de El País, contracarátula de La piedra alada, (Watanabe 2005). 


\section{Bibliografía}

Cuche, Denys, 1996, La notion de culture dans les sciences sociales, Paris, La Découverte, coll. «Repères».

Jara, Luis Fernando, 2007, «La representación del espacio en la poesía de José Watanabe. Una poética del ojo», Coloquio del GIS, «Territoires et sociétés dans les Amériques. Pratiques, cultures et représentations», 17-18 novembre 2007, Rennes.

Marco, Joaquín, El Cultural, (suplemento de El Mundo), www.elmundo.es, consultado el 30/12/2008.

Ordaz, Ramón, 2005, «Nuestro gran tema es la cotidianidad. Entrevista a José Watanabe», Poda, Revista Latinoamericana de Poesía, Mérida, Venezuela, Año 1, Núm. 1, Enero-Febrero, p. 41-52.

Matos Dias, Isabel, 2001, Merleau-Ponty. Une poiétique du sensible, Toulouse, Presses Universitaires du Mirail, coll. «Philosophica».

Takahashi Martinez, Yukyko, «Ciudadanos de origen japonés en el Perú durante la Segunda Guerra Mundial: una revisión de los debates actuales», http://www. discovernikkei.org., consultado el 08/03/2009.

Watanabe, José, 2008, Poesía completa, prólogo de Darío Jaramillo, Valencia, Pretextos.

Watanabe, José, 2005, La piedra alada, Valencia, Pre-Textos.

Watanabe, José, 2003, Elogio del refrenamiento, Antología poética, 1971-2003, edición y prólogo de Eduardo Chirinos, Sevilla, Renacimiento. 